\title{
EVALUACIÓN DEL RENDIMIENTO DEL CULTIVO DE PAPA BAJO LA APLICACIÓN DEL RIEGO DEFICITARIO (PRD) UTILIZANDO CINTAS DE RIEGO
}

\author{
EVALUATION OF POTATO CROP YIELD UNDER THE APPLICATION OF DEFICIT \\ IRRIGATION (PRD) USING IRRIGATION TAPES
}

\author{
${ }^{1}$ Miguel A. Sánchez D. \& ${ }^{2}$ Ruth E. Meza A.
}

\begin{abstract}
Resumen
El presente trabajo tiene como finalidad aplicar el riego deficitario de secado parcial de raíces (PRD) en el cultivo de papa, utilizando cintas de riego. El experimento se realizó en la parcela demostrativa del Departamento de Recursos Hídricos (DRH) de la Facultad de Ingeniería Agrícola, en la Universidad Nacional Agraria La Molina. Se trabajó dos tratamientos; un tratamiento testigo (T1) con una lámina de riego al 100\% de la necesidad hídrica del cultivo; y otro bajo el sistema de PRD (T2) con una lámina de 70\% de la necesidad hídrica del cultivo distribuidas en diseño completamente randomizado con cuatro repeticiones cada una, siendo las áreas de la pacerlas experimentales de 15 $\mathrm{m}^{2}$ por tratamiento haciendo un total de $60 \mathrm{~m}^{2}$ para el tratamiento T1 y $60 \mathrm{~m}^{2}$ para el tratamiento T2. Se determinaron los siguientes parámetros: volumen de agua total aplicado, altura de plantas, rendimiento $(\mathrm{Kg} / \mathrm{ha})$ total de tubérculos, número de tubérculos/planta, rendimiento de tubérculos por categoría, y eficiencia de uso de agua (EUA). Durante la campaña se hicieron en total 13 riegos en 2 etapas: la primera fue el período previo a la aplicación de tratamientos (fase no experimental) y la segunda fue la etapa de aplicación de tratamientos (fase experimental). La fase experimental empezó a los 51 días después de la siembra, cuando se hizo el primer riego experimental y se prolongó hasta el final de la campaña. El volúmen total de agua de riego aplicada durante el cultivo para el tratamiento 1 fue de $1857 \mathrm{~m}^{3}$ equivalente a una lámina de riego de $185.7 \mathrm{~mm}$. Para el tratamiento 2 fue de $1181 \mathrm{~m}^{3}$ equivalente a una lámina de riego de $118.1 \mathrm{~mm}$. La cosecha se realizó a los 107 días después de la siembra; y los rendimientos obtenidos fueron de 40.01 y $31.48 \mathrm{t} / \mathrm{ha}$ para el T1 y T2 respectivamente, existiendo diferencias altamente significativas a favor del tratamiento 1 . Al evaluar la Eficiencia de Uso de Agua, el PRD incrementó la eficiencia de uso de agua en $23.7 \%$.
\end{abstract}

Palabras clave: riego deficitario, secado parcial de raíces (PRD), papa, eficiencia de uso de agua (EUA).

\begin{abstract}
The The purpose of the present study is to apply in potato crops the Partial Rootzone drying (PRD) strategy using drip irrigation tapes in potato crops. The experiment was carried out in the demonstrative plots of the Department of Water Resources of the Faculty of Agricultural Engineering, La Molina National Agrarian University. Two treatments were evaluated: T1, Control group, with $100 \%$ of the water needed for the crop, and T2, the PRD system, with only $70 \%$ of the water needed for the crop. A Completely Ramdomized Design with two treatments and four replicates per treatment was used. The entire water volume applied, plant height, total tuber yield $(\mathrm{Kg} / \mathrm{ha})$, Number of tubers per plant, tuber yield per category, and efficiency of water use were determined. During the campaign, 13 irrigations were applied in two stages: the first one was the period before to the application of treatments (not experimental phase) and the second one was the stage of application of treatments (experimental phase). The experimental phase began 51 days after seeding, when the first experimental irrigation took place and it extended until the end of the campaign. The total volume of irrigation water applied during the campaign for $\mathrm{T} 1$ was $1857 \mathrm{~m}^{3}$ equivalently to an irrigation film of 185.7 $\mathrm{mm}$ and for T2 it was $1181 \mathrm{~m}^{3}$, equivalent to an irrigation film of $118.1 \mathrm{~mm}$. The harvest took place at 107 days after seeding and yields of 40.01 and $31.48 \mathrm{t} / \mathrm{ha}$ were obtained for the $\mathrm{T} 1$ and $\mathrm{T} 2$ treatments, respectively, existing highly significant differences in favor of T1. In assessing the water use efficiency, the PRD increased the efficiency of water use in $23.7 \%$.
\end{abstract}

Keys words: deficit irrigation, partial rootzone drying (PRD), potato, efficiency of water use.

\footnotetext{
${ }^{1}$ Profesor principal del Departamento de Recursos Hídricos. Facultad de Ingeniería Agrícola de la Universidad Nacional Agraria La Molina, Lima, Perú. Email: msanchez@lamolina.edu.pe

${ }^{2}$ Oficina Académica de Extensión y Proyección Social. Universidad Nacional Agraria La Molina, Lima, Perú.. E-mail: rmeza@lamolina.edu.pe
} 


\section{Introducción}

En la costa peruana la escasez de agua es uno de los problemas que afecta la producción agrícola, ocasionando limitaciones en la producción agropecuaria y la actividad productiva nacional. En estas condiciones se hace necesario reducir el consumo de agua limitando la cantidad aplicada, distribuyéndola con mayor eficiencia y evitando pérdidas innecesarias. El secado parcial de raíces (PRD) es una estrategia potencial en el ahorro de agua para riego; en este sistema se riega alternadamente sólo una parte del sistema radicular y su complemento se deja sin riego, hasta un determinado nivel de agotamiento de la humedad en el suelo.

También se puede decir que el PRD es una técnica de riego deficitario en donde una parte del sistema de las raíces permanece húmeda, con lo que se asegura un aporte hídrico limitado para mantener el vigor de la parte aérea, mientras que otra zona del sistema radical permanece seca, con lo que se liberan señales bioquímicas que son transportadas vía xilema a las hojas, que una vez en la parte aérea regulan la apertura de estomas y limitan las pérdidas de agua vía transpiración. Rojas, 2007, evaluó en condiciones de campo el efecto del PRD en el cultivo de papa, experimento realizado en el Centro Internacional de la Papa, en el cual comparó el PRD con el riego normal (RN) y con el sistema de riego por gravedad. Se aplicaron cuatro tratamientos de riego en surcos alternos: dos de RN y dos de PRD; al 100\% y 50\% de la lámina de riego requerida por el cultivo. Los resultados mostraron que las diferencias debidas a la lámina de riego fueron mucho más notorias en el RN que en el PRD. Mientras que al comparar las técnicas de riego, el rendimiento de tubérculo fue mayor en el RN que en el PRD, la EUA no arrojó diferencias significativas y en cuanto a la calidad, y la mayoría de parámetros de crecimiento evaluados el PRD mostró características ligeramente superiores al RN, destacando el efecto positivo que tuvo el PRD en el desarrollo radicular, logrando características de adaptación al estrés hídrico muy favorables en la papa.

El presente el trabajo tuvo como objetivo evaluar el rendimiento y cuantificación del volumen de agua aplicado en el cultivo de papa variedad única bajo condiciones de suelo y clima de costa aplicando el riego deficitario de secado parcial de raíces (PRD), utilizando el sistema de riego por goteo (cintas de riego) y la determinación de la eficiencia de uso e agua (EUA).

\section{Materiales y Métodos}

El experimento fue realizado en la parcela demostrativa del Departamento de Recursos de Recursos Hídricos $(\mathrm{DRH})$ de la Facultad de Ingeniería Agrícola, en la Universidad Nacional Agraria La Molina a una altitud de 238 m.s.n.m., $12^{\circ} 05^{\prime} 06^{\prime \prime}$ de latitud sur y $76^{\circ} 57^{\prime} 00^{\prime \prime}$ de longitud oeste, durante los meses de Agosto a Diciembre del 2008.

\section{Material Vegetal}

La variedad de papa con la que se trabajó fue UNICA, por ser apropiada para el consumo en fresco, y presentar tolerancia al calor pudiendo tuberizar con temperaturas nocturnas de hasta $16^{\circ} \mathrm{C}$. La siembra se llevó a cabo el 21 de Agosto de 2008. De acuerdo al análisis de suelos la dosis de fertilización aplicada de NPK fue de 120 $100-100$, correspondiente a una cantidad de $3.13 \mathrm{Kg}$ de Urea, $2.61 \mathrm{Kg}$ de Superfosfato triple y $2 \mathrm{Kg}$ de cloruro de potasio. La fertilización fue en dos momentos: en la siembra se aplicó la mitad del nitrógeno (úrea) y el $100 \%$ del fósforo (superfosfato triple) y el potasio (sulfato de potasio). La mitad restante de nitrógeno se aplicó en el aporque. El control fitosanitario fue cultural y químico, con aplicaciones de productos químicos para el control de plagas y enfermedades y las malezas se controlaron manualmente. Desde el inicio hasta el final del experimento se irrigó con el agua proveniente del río Rímac a través del canal Ate, el que se deriva hacia el área experimental mediante canales revestidos, para ser almacenada en un reservorio de $20 \mathrm{~m}^{3}$. Seguidamente el agua ingresa al sistema de riego a través de la red primaria y laterales respectivos.

\section{Tratamientos}

El diseño estadístico empleado en el desarrollo del trabajo de investigación fue el Diseño Completamente Randomizado con cuatro repeticiones cada uno. Un tratamiento testigo (T1) con una lámina de riego al 100\% de la necesidad hídrica del cultivo; y otro bajo el sistema de PRD (T2) con una lámina de $70 \%$ de la necesidad hídrica del cultivo. Los datos se analizaron con el programa de cómputo utilizado para efectuar los análisis estadístico Statistical Analysis System (SAS).

El área neta de trabajo fue de $120 \mathrm{~m}^{2}$ la cual se dividió en 8 parcelas experimentales de $15 \mathrm{~m}^{2}$ cada una. Cada unidad experimental conformada por 3 líneas de riego de 5 metros de longitud, 12 correspondiente al Tratamiento Testigo (T1) y 24 correspondiente al PRD (T2). Para el caso del tratamiento testigo se instaló por hilera de plantas un lateral de riego a una distancia de $10 \mathrm{~cm}$ de la planta, en el PRD se instaló por hilera de plantas dos laterales de riego espaciados $0.20 \mathrm{~m}$ y emisores distribuidos cada $0.30 \mathrm{~m}$ en cada lateral. Cada línea de riego se operó de manera individual lo que permitió alternar las aplicaciones de riego entre la parte izquierda y la parte derecha del sistema radical, tal como se indica en las Figuras 1 y 2. El caudal de los emisores de la cinta de riego fue de 11/h. El sistema permite dotar de agua a cada unidad experimental independientemente. Con la finalidad de controlar el volumen de agua aplicado en cada parcela se instaló un caudalímetro. Para el control de la presión de trabajo del sistema de riego se contó con un manómetro instalado al inicio de todo el sistema de distribución. El riego fue programado a partir de los datos climáticos de la estación Alexander Von Humbolt de la UNALM. 


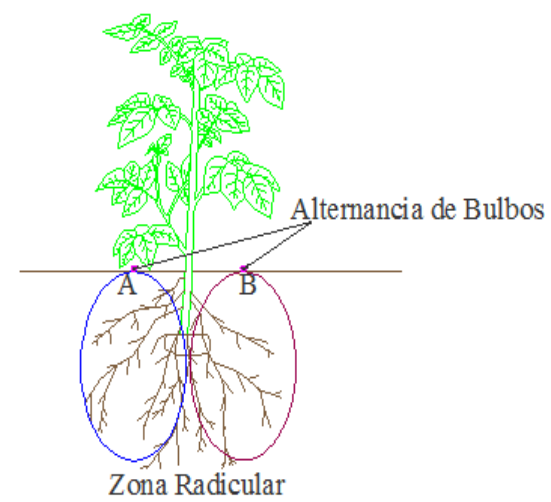

Figura 1. Vista Perfil de la alternancia de aplicación de agua a la planta. riegos se hicieron de manera uniforme en todo el campo ya que el objetivo era lograr que las plantas alcancen un desarrollo vegetativo y radicular uniforme y adecuado para la posterior etapa de aplicación de tratamientos. El régimen hídrico del suelo durante esta fase está representado en la Figura 3. Los picos de la curva indican los días en los que se realizaron los riegos.

La fase experimental empezó a los 51 días después de la siembra, cuando se hizo el primer riego experimental y se prolongó hasta el final de la campaña. Para determinar el contenido de humedad se empleó el método gravimétrico y el método del tensiómetro en la fase no experimental y experimental respectivamente. En total se hicieron 7 riegos experimentales, que se diferenciaron en lámina de agua (al 100 y 70\%) a través del riego por goteo.

Humedad del suelo en el campo durante la fase no experimental

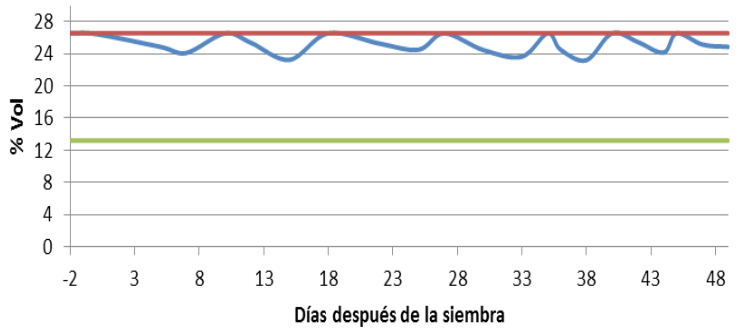

Figura 3. Humedad del suelo durante la fase no experimental.

La cosecha se realizó a los 107 días (06 de diciembre de 2008) después de la siembra; los rendimientos obtenidos están referidos en base a un surco central cosechado en cada unidad experimental.

\section{Evaluaciones experimentales \\ Del Cultivo}

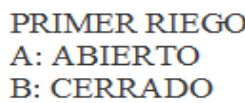

\section{B: SEGUNDO RIEGO \\ A: CERRADO \\ B: ABIERTO}

Figura 2. Vista en planta de la alternancia de aplicación de agua a la planta.

\section{Contenido de Humedad}

Durante la campaña se hicieron en total 13 riegos en 2 etapas de riego: la primera fue el período previo a la aplicación de tratamientos (fase no experimental) y la segunda fue la etapa de aplicación de tratamientos (fase experimental).

La fase no experimental empezó desde que se hizo el primer riego, 3 días antes de la siembra, con la finalidad de humedecer el suelo para favorecer la brotación de los tubérculos. Luego, se hicieron 6 riegos de acuerdo al régimen establecido cada 5 días. Durante esta fase, los

\section{A. Evaluación del Crecimiento y Desarrollo Emergencia de Plantas}

Para evaluar la emergencia de plantas se realizó observaciones en 3 fechas diferentes; lográndose $99 \%$ de emergencia a los 26 días después de la siembra.

\section{Altura de plantas}

Se midió desde el cuello de la planta hasta el final del tallo principal. Para evaluar el crecimiento de las plantas se realizó las mediciones de altura en el surco central de cada unidad experimental y se tomó el promedio de los datos obtenidos. 


\section{B. Evaluaciones a la Cosecha}

\section{Rendimiento (Kg/ha)Total de Tubérculos}

Peso Total por hectárea: Es el valor estimado llevado a hectárea con relación al valor total hallado para cada unidad experimental.

Para cada tratamiento de riego se ha considerado el producto final cosechado.

$$
\text { Rdto }(\mathrm{Kg} / \mathrm{ha})=\frac{\text { Peso } \times \text { Parcela }(\mathrm{Kg})}{\text { Area de la parcela }\left(\mathrm{m}^{2}\right)} \times 10000 \mathrm{~m}^{2}
$$

\section{Número de tubérculos/planta}

Para evaluar el número de tubérculos se realizó el conteo por planta en el surco central de cada unidad experimental, luego se tomó el promedio de los datos obtenidos.

\section{Rendimiento de Tubérculos por Categoría}

Se tomó los datos de cada surco central y se clasificó de acuerdo a las categorías establecidas por el CIP. Esta clasificación se hizo en base al peso de cada tubérculo y se muestra en el cuadro 1.

Cuadro 1. Clasificación del rendimiento de tubérculos por categoría.

\begin{tabular}{lc}
\hline \multicolumn{1}{c}{ Categoría } & Peso (gr) \\
\hline Extra & $>=266$ \\
Selecta & $135-265$ \\
Comercial & $86-134$ \\
Doméstica & $37-85$ \\
Baby & $21-36$ \\
Otros & $<20$ \\
\hline
\end{tabular}

\section{Del Sistema de Riego}

- Presión Promedio de trabajo del sistema: Se evaluó durante todo el ciclo vegetativo del cultivo de papa.

- Caudal: se evaluó a la siembra y al final del ciclo vegetativo del cultivo.

- Coeficiente de Uniformidad del sistema de riego a la siembra del cultivo.

\section{Parámetro Agronómico}

Eficiencia de uso de agua $\left(\mathbf{K g} / \mathbf{m}^{3}\right)$ : se entiende en el presente trabajo por eficiencia de uso de agua EUA, al rendimiento de tubérculos obtenidos por cada metro cúbico de agua aplicado.

$$
\operatorname{EUA}\left(\mathrm{Kg} / \mathrm{m}^{3}\right)=\frac{\text { Rendimiento de tubérculos comerciales }\left(\frac{\mathrm{kg}}{\mathrm{Ha}}\right)}{\text { Vol.de Agua Aplicado }\left(\frac{\mathbf{m}^{3}}{\mathrm{Ha}}\right)}
$$

\section{Resultado y discusión}

\section{Necesidad de agua del cultivo}

Para el cálculo de las necesidades de agua del cultivo de papa se hizo uso del programa diseñado por la FAO denominado CROPWAT, el cual permitió obtener además de las necesidades de agua del cultivo, las fechas y número de riegos.

\section{Evapotranspiración de referencia Eto según Penman- Monteith}

Haciendo uso del método Penman-Monteith, a través del programa CROPWAT, se determinó la ETP del cultivo de referencia el cual se muestra en el cuadro 2, cuyos valores están comprendidos entre 1.95 (agosto) y 3.46 (noviembre) $\mathrm{mm} / \mathrm{día}$.

El valor de la precipitación para todos los meses se consideró nulo.

Cuadro 2. Valores de Etp (mm/día).

\begin{tabular}{lc}
\hline Mes & Etp $(\mathrm{mm} /$ día $)$ \\
\hline Agosto & 1.95 \\
Setiembre & 2.53 \\
Octubre & 3.05 \\
Noviembre & 3.46 \\
\hline
\end{tabular}

\section{Factor de cultivo Kc, Pr, F y Ky}

Para el cultivo de papa variedad única se tomó un período vegetativo de 105 días, los valores de duración, Kc, profundidad de raíz, nivel de agotamiento y respuesta al rendimiento de cada fase de desarrollo del cultivo se muestran en el cuadro 3 .

Cuadro 3. Datos del cultivo de papa.

\begin{tabular}{lllll}
\hline Etapas de crecimiento & Inicial & Desarrollo & Media & Final \\
\hline Duración (días) & 25 & 30 & 30 & 20 \\
Coeficiente Kc & 0.45 & - & 1.15 & 0.85 \\
Profundidad Raíz (m) & 0.10 & - & 0.30 & 0.30 \\
Nivel de Agotamiento & 0.25 & - & 0.25 & 0.25 \\
Respuesta al rend. Ky & 0.45 & 0.80 & 0.70 & 0.20 \\
\hline
\end{tabular}

Fuente: F. Yagué (1996); Técnicas de Riego.

Asimismo se tomó en cuenta los siguientes valores que se muestran en el cuadro 4

Cuadro 4. Características del suelo.

\begin{tabular}{lc}
\hline \multicolumn{1}{c}{ Tipo de Suelo } & Franco Arenoso \\
\hline Humedad Disponible del suelo $(\mathrm{mm} / \mathrm{m})$ & 140 \\
Profundidad radicular máxima $(\mathrm{mm})$ & $0.30 \mathrm{~m}$ \\
$\begin{array}{l}\text { Humedad inicial disponible en el suelo } \\
(\mathrm{mm} / \mathrm{m})\end{array}$ & 140 \\
\hline
\end{tabular}

Se estableció como intervalos de riego cada 5 días, llegando en cada riego hasta la capacidad de campo. 


\section{Cantidad de agua aplicada}

En el cuadro 5 se observa las fechas de los riegos y volumen de agua aplicada en cada tratamiento durante la campaña; y está representado en la Figura 4. El número de riegos durante la fase no experimental fueron seis los que se caracterizaron por ser homogéneos y los cuales se dieron con intervalos de 5-9 días.

En el cuadro 6 se observa el volumen de agua aplicada en cada tratamiento durante la campaña. Como se ve, durante la fase no experimental para el caso del tratamiento testigo (T1) se aplicó menor cantidad de agua que en la fase experimental, ya que en la fase experimental que corresponde desde la tuberización hasta las madurez completa el requerimiento hídrico es mayor (Egúsquiza, 2000), a diferencia del Tratamiento PRD que durante la fase experimental se aplicó el $70 \%$ de la lámina aplicada al T1, esto por ser objetivo de evaluación de este experimento. Sin embargo, el volumen total de agua aplicado durante la campaña en el tratamiento testigo (T1) es aceptable, comparado con (Villa, 1998) que menciona que, empleando el riego por goteo se obtiene un ahorro de agua del 50\% con respecto al riego por gravedad; además (Rojas, 2007), señala que la cantidad de agua utilizado por el cultivo de papa, empleando el sistema de riego por gravedad es de 3500 a 5000 metros cúbicos por hectárea de terreno cultivado.

En total la cantidad de agua aplicada al cultivo de papa para el T1 bajo el sistema de riego por goteo fue de $\mathbf{1 8 5 7}$ $\mathbf{m}^{3} / \mathbf{h a}$; comparando este resultado con los autores citados líneas arriba, se obtiene un ahorro de agua equivalente a $2393.00 \mathrm{~m}^{3} / \mathbf{h a}$, es decir la cantidad de agua requerida por el cultivo representa el $\mathbf{4 3 . 7 0 \%}$ del volumen total de agua utilizando el riego por gravedad, durante la campaña (03 meses). Para el T2 la cantidad de agua aplicada al cultivo de papa bajo el sistema de riego por goteo fue de $\mathbf{1 1 8 1}$ $\mathbf{m}^{3} / \mathbf{h a}$, que comparado con el T1 representa un ahorro de agua equivalente a $676 \mathrm{~m}^{3} / \mathrm{ha}$, es decir la cantidad de agua requerida por el cultivo representa el $64 \%$ del volumen total de T1.

Cuadro 5. Fecha de riego y volúmenes de agua aplicada cada tratamiento.

\begin{tabular}{lcc}
\hline \multirow{2}{*}{ Fecha } & \multicolumn{2}{c}{ Tratamientos } \\
\cline { 2 - 3 } & T1 & T2 \\
\hline $31 / 08 / 2008$ & 0.33 & 0.33 \\
$08 / 09 / 2008$ & 0.35 & 0.35 \\
$17 / 09 / 2008$ & 0.54 & 0.54 \\
$25 / 09 / 2008$ & 0.66 & 0.66 \\
$30 / 09 / 2008$ & 0.79 & 0.79 \\
$05 / 10 / 2008$ & 0.93 & 0.93 \\
\hline Fase no experimental $\left(\mathrm{m}^{3}\right)$ & 3.59 & 3.59 \\
\hline $11 / 10 / 2008$ & 1.08 & 0.76 \\
$20 / 10 / 2008$ & 1.21 & 0.42 \\
$27 / 10 / 2008$ & 1.25 & 0.44 \\
$02 / 11 / 2008$ & 1.33 & 0.47 \\
$08 / 11 / 2008$ & 1.37 & 0.48 \\
\hline
\end{tabular}

Continuación del cuadro 5

\begin{tabular}{lcc}
\hline $13 / 11 / 2008$ & 1.29 & 0.47 \\
$18 / 11 / 2008$ & 7.55 & 3.49 \\
\hline Fase experimental $\left(\mathrm{m}^{3}\right)$ & 11.14 & 7.09 \\
\hline Total Aplicado $\left(\mathrm{m}^{3}\right)$ & & \\
$\mathrm{N}^{0}$ de Riegos & 6 & 6 \\
Uniformes & 6 & 7 \\
Diferenciados & 12 & 13 \\
Total & & \\
\hline
\end{tabular}

\section{VOLUMEN DE AGUA APLICADO EN CADA RIEGO AL} CULTIVO DE PAPA - VARIEDAD UNICA

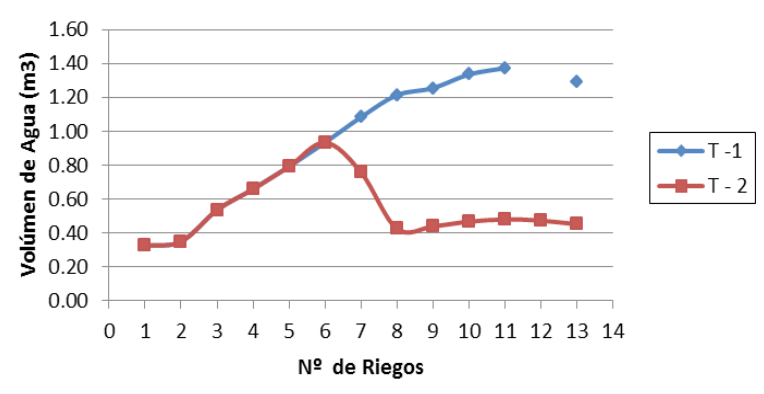

Figura 4. Volumen de agua aplicado en cada riego al cultivo de papa-variedad Única.

Cuadro 6. Volumen de agua aplicada $\left(\mathrm{m}^{3} / \mathrm{ha}\right)$ en cada tratamiento por etapa y campaña.

\begin{tabular}{cccc}
\hline Tratamiento & $\begin{array}{c}\text { Fase no } \\
\text { experimental }\end{array}$ & $\begin{array}{c}\text { Fase } \\
\text { experimental }\end{array}$ & $\begin{array}{c}\text { Volumen total } \\
\text { por campaña }\end{array}$ \\
\hline T1 & 599 & 1258 & 1857 \\
T2 & 599 & 582 & 1181 \\
\hline
\end{tabular}

\section{Altura de planta}

La variación de altura de planta de los tratamientos muestra una tendencia exponencial. Las plantas sometidas al tratamiento $\mathrm{T} 1$ mostraron una mayor intensidad de crecimiento entre los 50 y 65 días después de la siembra, alcanzando su máxima altura promedio de $90.18 \mathrm{~cm}$ a los 75 días, mientras las plantas sometidas al tratamiento $\mathrm{T} 2$ crecieron con mayor intensidad hasta los 55 días, alcanzando su máxima altura promedio de $72.65 \mathrm{~cm}$ a los 65 días, fecha a partir de la cual empezó a disminuir. Para la variable altura de planta se detectaron diferencias altamente significativas $(\mathrm{P}<=0.05)$ entre los dos tratamientos, siendo mayor T1 que T2. Es decir, el riego con suministro normal de agua resultó ser más favorable que el riego parcial. En los Cuadros 7 y 8 se presentan los análisis de variancia y de medias.

Cuadro 7. Análisis de variancia de altura de planta del cultivo de papa sometido a dos tratamientos de riego.

\begin{tabular}{lll}
\hline \multicolumn{1}{c}{ Fuente } & \multicolumn{1}{c}{ GL } & \multicolumn{1}{c}{ CM } \\
\hline Tratamientos & 1 & $614.2512500 * *$ \\
Error Experimental & 6 & 0.4962500 \\
Error Total & 7 & \\
\hline
\end{tabular}

* Significativo al 0.05 de probabilidad

** Significativo al 0.01 de probabilidad

$\mathrm{CV}=0.865285 \%$ 
Cuadro 8. Comparación de medias de altura de planta del cultivo de papa sometido a dos tratamientos de riego según la prueba de Tukey a nivel de $\mathrm{p}=0.05$.

\begin{tabular}{lll}
\hline Tratamiento & Altura de planta $\mathrm{cm}$ \\
\hline Testigo T1 & 90.1750 & $\mathrm{~A}$ \\
Riego parcial T2 & 72.65 & $\mathrm{~B}$ \\
\hline
\end{tabular}

Efectos sobre el rendimiento total, número de tubérculos por planta y rendimiento de tubérculos por categoría.

\section{Rendimiento Total}

En el cuadro 9 se presentan los resultados del ANVA para los rendimientos totales de tubérculos de los tratamientos en estudio. Dichos resultados indican que existen diferencias altamente significativas entre ambos tratamientos en estudio. Por ello, en el cuadro 10 se presenta los resultados de la prueba de comparación de medias de Tukey, a nivel de $\mathrm{p}=0.05$ para los tratamientos de riego.

Los rendimientos obtenidos fueron 40010 y $31480 \mathrm{Kg} / \mathrm{ha}$, para T1 y T2 respectivamente. Como se puede observar el tratamiento testigo (T1), fue mejor en comparación con el riego parcial (T2). Resultado similar fue obtenido por (Guliver, 2007) quien al estudiar la producción del cultivo de papa variedad UNICA detectó que el rendimiento de tubérculos fue menor en el PRD como consecuencia de la disminución de la lámina, $45000 \mathrm{Kg} / \mathrm{ha}$ para una lámina del $100 \%$ Etc y $31000 \mathrm{Kg} /$ ha para una lámina del $50 \%$ de $\mathrm{T} 1$ utilizando riego por gravedad, valores que se encuentran en el rango a lo obtenido en el experimento de papa variedad única empleando riego por goteo.

Con ello se corrobora lo afirmado por (Egúsquiza, 2000) quien afirma que existe una relación positiva y directa entre la cantidad de agua que dispone la planta y el rendimiento comercial.

Cuadro 9. Análisis de variancia del rendimiento total del cultivo de papa sometido a dos tratamientos de riego.

\begin{tabular}{lcl}
\hline & GL & CM \\
\hline Tratamientos & 1 & $145521800.0 * *$ \\
Error Experimental & 6 & 943133.3 \\
Error Total & 7 & \\
* Significativo al 0.05 de probabilidad & \\
** Significativo al 0.01 de probabilidad & $\mathrm{CV}=2.716885 \%$
\end{tabular}

Cuadro 10. Comparación de medias del rendimiento total del cultivo de papa sometido a dos tratamientos de riego según la prueba de Tukey a nivel de $\mathrm{p}=0.05$.

\begin{tabular}{ll}
\hline Tratamiento & \multicolumn{2}{l}{ Rendimiento total Kg/ha } \\
\hline Testigo T1 & $40010.00 \mathrm{~A}$ \\
Riego parcial T2 & $31480.00 \mathrm{~B}$ \\
\hline
\end{tabular}

\section{Número de Tubérculos por planta}

En el cuadro 11, se presentan los resultados del ANVA para el número promedio de tubérculos por planta. Los resultados nos indican que existen diferencias significativas para los tratamientos en estudio. Los números de tubérculos cosechados por planta para los tratamientos T1 y T2 fueron 13.7250 y 10.7250 respectivamente. De ello se deduce que para la producción obtenida de papa, el número de tubérculos por planta se incrementa a medida que aumenta la lámina de riego.

Cuadro 11. Análisis de variancia del número de tubérculos por planta $(\mathrm{N}$ Tub /pl) del cultivo de papa sometido a dos tratamientos de riego.

\begin{tabular}{lcl}
\hline \multicolumn{1}{c}{ Fuente } & GL & \multicolumn{1}{c}{ CM } \\
\hline Tratamientos & 1 & $18.0000000^{*}$ \\
Error Experimental & 6 & 1.57916667 \\
Error Total & 7 & \\
* Significativo al 0.05 de probabilidad & \\
** Significativo al 0.01 de probabilidad & \\
CV $=10.27934 \%$ & &
\end{tabular}

En el cuadro 12 se presenta la comparación de medias del número de tubérculos por planta (N Tub/pl) del cultivo de papa sometido a dos tratamientos de riego según la prueba de Tukey.

Cuadro 12. Comparación de medias del número de tubérculos por planta $(\mathrm{N} \mathrm{Tub} / \mathrm{pl})$ del cultivo de papa sometido a dos tratamientos de riego según la prueba de Tukey a nivel de $\mathrm{p}=0.05$.

\begin{tabular}{lc}
\hline \multicolumn{1}{c}{ Tratamiento } & $\begin{array}{c}\text { Número de tubérculos por } \\
\text { planta }(\mathrm{N} \mathrm{Tub} / \mathrm{pl})\end{array}$ \\
\hline Testigo T1 & $13.7250 \mathrm{~A}$ \\
Riego parcial T2 & $10.7250 \quad \mathrm{~B}$ \\
\hline
\end{tabular}

\section{Rendimiento de Tubérculos por categoría}

En el cuadro 13 se muestran los resultados del ANVA para los rendimientos promedio de tubérculos, de acuerdo al peso alcanzado, clasificados como categorías extra, selecta, comercial, doméstica, baby y otros. La fig. 5 nos permite apreciar el comportamiento de dichas categorías. El rendimiento de tubérculos para las categorías extra, selecta, doméstica, baby y otros no muestran diferencias significativas, mientras que para la categoría comercial existen diferencias significativas. En el cuadro 14 la prueba de Tukey. 
Cuadro 13. Análisis de variancia del número de tubérculos por planta del cultivo de papa sometido a dos tratamientos de riego.

\begin{tabular}{|c|c|c|c|c|c|c|c|}
\hline Fuentes & $\begin{array}{c}\text { Grados de } \\
\text { Libertad }\end{array}$ & $\begin{array}{c}\text { Cuadrados } \\
\text { Medios } \\
\text { Rendimiento } \\
\text { Extra }\end{array}$ & $\begin{array}{c}\text { Cuadrados } \\
\text { Medios } \\
\text { Rendimiento } \\
\text { Selecta }\end{array}$ & $\begin{array}{c}\text { Cuadrados } \\
\text { Medios } \\
\text { Rendimiento } \\
\text { Comercial }\end{array}$ & $\begin{array}{l}\text { Cuadrados } \\
\text { Medios } \\
\text { Rendimiento } \\
\text { Doméstica }\end{array}$ & $\begin{array}{c}\text { Cuadrados } \\
\text { Medios } \\
\text { Rendimiento } \\
\text { Baby }\end{array}$ & $\begin{array}{l}\text { Cuadrados } \\
\text { Medios } \\
\text { Rendimiento } \\
\text { Otros }\end{array}$ \\
\hline Tratamientos & 1 & $3726450 \mathrm{NS}$ & $5024450 \mathrm{NS}$ & $12500000 *$ & $13624200 \mathrm{NS}$ & 500000. & $1800.00000 \mathrm{NS}$ \\
\hline Error Experimental & 6 & 3316050 & 3936916.67 & 1299066.67 & 4234066.67 & 903866.667 & 7666.66667 \\
\hline Error Total & 7 & & & & & & \\
\hline $\mathrm{CV}(\%)$ & & $20.24 \%$ & $20.80 \%$ & $15.00 \%$ & $31.20 \%$ & $39.61 \%$ & $14.24 \%$ \\
\hline
\end{tabular}

Ns=No significativo

* Significativo al 0.05 de probabilidad

**Significativo al 0.01 de probabilidad

Cuadro 14. Comparación de medias del número de tubérculos por planta del cultivo de papa sometido a dos tratamientos de riego según la prueba de Tukey a nivel de $\mathrm{p}=0.05$.

\begin{tabular}{lccc}
\hline \multicolumn{1}{c}{ Variable } & Tratamiento T1 & Tratamiento T2 & Significación \\
\hline Rendimiento extra & $9680 \mathrm{~A}$ & $8315 \mathrm{~A}$ & NS \\
Rendimiento selecta & $10330 \mathrm{~A}$ & $8745 \mathrm{~A}$ & NS \\
Rendimiento comercial & $8850 \mathrm{~A}$ & $6350 \mathrm{~A}$ & Significativo \\
Rendimiento domestica & $7900 \mathrm{~A}$ & $5290 \mathrm{~A}$ & $\mathrm{NS}$ \\
Rendimiento Baby & $2650 \mathrm{~A}$ & $2150 \mathrm{~A}$ & $\mathrm{NS}$ \\
Rendimientos otros & $600 \mathrm{~A}$ & $630 \mathrm{~A}$ & $\mathrm{NS}$ \\
\hline
\end{tabular}

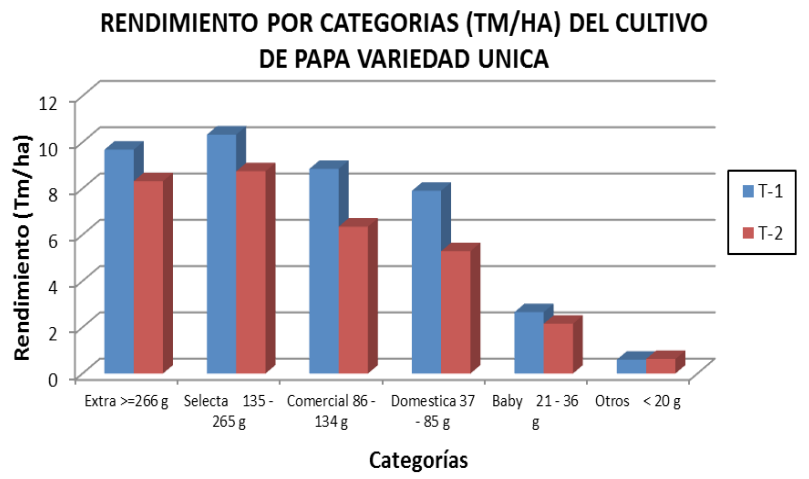

Figura 5. Volumen de agua aplicado en cada riego a l cultivo de papa-variedad Única.

\section{Eficiencia de Uso de Agua}

El cuadro 11, muestra los resultados obtenidos de la Eficiencia de Uso de Agua (EUA) del cultivo de papa. Asimismo se aprecia que existe diferencias altamente significativas entre ambos tratamientos en estudio. Los resultados de la prueba de comparación de Tukey, a nivel de $p=0.05$ para los tratamientos de riego se muestran en el cuadro 30.

Los valores obtenidos de EUA fueron de 26.6450 y 21.5450, para los tratamientos T2 y T1 respectivamente. Con ello se demuestra que la EUA fue significarivamente mejorada, en $23.7 \%$, en aquellas plantas expuestas bajo el sistema de $\mathrm{PRD}$, en relación con aquellas bajo riego por goteo al $100 \%$ Etc. La EUA, se expresa como la producción de papa en Kilogramos por metro cúbico de agua aplicado al cultivo, siendo este valor menor cuando se aplica mayor lámina de riego (T1) y mayor para una menor lámina de riego (T2).
Cuadro 11. Análisis de variancia de eficiencia de uso de agua del cultivo de papa sometido a dos tratamientos de riego.

\begin{tabular}{lcl}
\hline Fuente & GL & CM \\
\hline Tratamientos & 1 & 52.02000000 \\
Error Experimental & 6 & 0.28176667 \\
Error Total & 7 & \\
* Significativo al 0.05 de probabilidad & \\
** Significativo al 0.01 de probabilidad & \\
CV $=2.203017 \%$ &
\end{tabular}

\section{Conclusiones}

- Durante el ensayo se dieron dos tipos de riego, riegos uniformes y riegos diferenciados; aplicándose 6 riegos uniformes con la finalidad de permitir el establecimiento del cultivo y seguidamente se dio inició a los tratamiemtos, habiéndose aplicado 6 y 7 riegos para el tratamiento 1 y 2 respectivamente.

- El volúmen total de agua de riego aplicada durante el cultivo para el tratamiento 1 fue de $1857 \mathrm{~m}^{3} /$ ha equivalente a una lámina de riego de $185.7 \mathrm{~mm}$. Para el tratamiento 2 fue de $1181 \mathrm{~m}^{3} /$ ha equivalente a una lámina de riego de $118.1 \mathrm{~mm}$.

- Los rendimientos obtenidos a la cosecha para el T1 y T2 fueron de 40.01 y 31.48 t/ha respectivamente, observándose el mayor rendimiento para el tratamiento 1, existiendo diferencias altamente significactivas entre ambos tratamientos.

- El PRD recibió riegos alternos uno al lado derecho y otro al lado izquierdo cada 5-9 días.

- Los números de tubérculos cosechados promedio por planta para los tratamientos T1 y T2 fueron 13.7250 y 10.7250 respectivamente, observándose un mayor número de tubérculos para el tratamiento 1, existiendo diferencias significativas para los tratamientos 
en estudio. Del mismo se detectaron diferencias altamente significativas para la variable altura de las planta, siendo los valores obtenidos de 90.18 y 72.65 $\mathrm{cm}$. para $\mathrm{T} 1$ y $\mathrm{T} 2$ respectivamente.

- Al evaluar la Eficiencia de Uso de Agua, el PRD incrementó la eficiencia de uso de agua en $23.7 \%$. Así, el PRD muestra potencial para el ahorro hídrico, importante para regiones agroecológicos con limitaciones de agua.

- Finalmente, el sistema de riego localizado de alta frecuencia, dada sus particulares características permitió manejar y controlar eficientemente los regímenes de riego en estudio.

\section{Recomendaciones}

- Efectuar investigaciones para las diferentes variedades de papa en la costa y sierra.

- Realizar nuevas investigaciones con el método de riego utilizado en diferentes épocas del año, otras zonas y en otras variedades.

- Realizar trabajos que aseguren una división más precisa del sistema radicular donde se tenga un mejor control de la humedad del suelo.

- Tomar en cuenta que el PRD reduce el período de cosecha en los cultivos.

- Se recomienda seguir la investigación con volúmenes de agua mayores a los utilizados en el presente estudio.

- Realizar en nuevas investigaciones el análisis económico para determinar la rentabilidad y la relación beneficio costo.

\section{Literatura citada}

Alarcón, J.J.; Vera, J.; Mounzer, O.; Torrecillas A.; García-Orellana, Y.; Nicolás, E. y Magaña, I. 2006. Estrategias de Riego Deficitario en Melocotonero Temprano. Vida Rural. (ISSN) 225:28-32.

Doorenbos, J. y Kassan, H. 1979. Efecto del Agua sobre el rendimiento de los cultivos. FAO: Riego y Drenaje Publicación N ³3 Roma -Italia. Pág. 163-166.

Egusquiza, B. R. 2000. La Papa. Producción, Transformación y Comercialización. Primera edición. Lima, Perú, 192 p.

Fuentes, Y. J. 2003. Técnicas de Riego. Ediciones Mundi Prensa, Sexta Edición. Madrid, España. Pág. 286-287.

Garcia, I. y Briones, G. 2007. Sistemas de Riego por Aspersión y Goteo. Ediciones Trillas, México. Segunda edición $182 \mathrm{p}$.

Levitt, J. 1980. Responses of Plants to Environmental Stress. Vol. I. Chilling, and High Temperature Stresses. Academic Pres, New York.

Mamani, R. F. 1993. Comportamiento Morfológico de dos Clones de Papa sometidos a Estrés Hídrico por Sequía. Tesis para optar el Grado de Magíster Scientiae. UNALM. Lima- Perú.
Medina, J. 1981. Riego por Goteo. Teorìa y Práctica. Ediciones Mundi Prensa, Madrid. Segunda Edición revisada. $216 \mathrm{p}$.

Vermeiren, L. y Joblin, G. 1986. "Riego Localizado" FAO: Riego y Drenaje N³6.Madrid-España.Pág.8-9.

Shahnazari, A.; Andersen, M. N.; Liu, F.; Jacobsen, S.-.E. and Jensen, C. R. 2008. Partial Root Zone Drying (PRD) Sustains Yield Of Potatoes (Solanum Tuberosum L.) at Reduced Water Supply. Acta Hort. (ISHS) 792:581586. http://www.actahort.org/books/792/792 68.htm.

Vasquez, V. A. y Chang, N. L. 1992. El Riego. Principios Básicos. Tomo I. Lima, Perú, 192 p.

Zegbe, J. A.; Behboudian, M. H.; Clothier, B. E. Response of Processing Tomato to Partial Rootzone Irrigation. 2007. Terra Latinoamericana. (ISSN) 25:6167. http://redalyc.uaemex.mx/src/inicio/ArtPdfRed. jsp? $\mathrm{iCve}=57311513008$ 